\title{
EFECTO DEL FUNGICIDA IPRODIONE Y SUS MEZCLAS CON THIRAM Y TRITICONAZOLE EN EL CONTROL DE Drechslera teres EN SEMILLAS DE CEBADA
}

\author{
MARCELO A. CARMONA ${ }^{1}$, DORA E. BARRETO ${ }^{1,2}$ \& ERLEI M. REIS ${ }^{3 .}$
}

'Fitopatología. Facultad de Agronomía. Universidad de Buenos Aires. Av. San Martín 4453 (1417), Capital Federal, Argentina. e-mail: macarmona@ sion.com; ${ }^{1,2}$ Instituto de Fitopatología y Fisiología Vegetal, INTA, Castelar; ${ }^{3}$ Faculdade de Agronomía, Medicina e Veterinaria, Universidade de Passo Fundo, Cx. Postal 566 e 567, 99001-970, Passo Fundo RS, Brasil

(Aceito para publicação em 21/03/2001)

Autor para correspondência: Marcelo A. Carmona

CARMONA, M., BARRETO, D. \& REIS, E. M. Efecto del fungicida iprodione y sus mezclas con thiram y triticonazole en el control de Drechslera teres en semillas de cebada. Fitopatología Brasileira 26:176-179. 2001.

\section{RESUMEN}

La mancha en red de la cebada (Hordeum vulgare) es la enfermedad más importante de este cultivo en la Argentina. El tratamiento químico de semillas es una medida importante de control para impedir la introducción y dispersión de Drechslera teres. Los objetivos de este trabajo fueron 1) evaluar la fungitoxicidad específica in vitro de diferentes dosis del fungicida iprodione y sus mezclas con thiram y triticonazole y compararlos con los fungicidas más utilizados (flutriafol y tebuconazole), y 2) valorar a campo, el mejor tratamiento obtenido in vitro. Los resultados mostraron que los fungicidas actualmente en uso en Argentina fueron menos eficientes en el control de $D$. teres que el iprodione sólo (50 g i.a.) o en mezcla con triticonazole $\mathrm{y}$ thiram. En parcelas apareadas con iprodione + triticonazole versus testigo se evaluaron el número de focos de infección primaria, incidencia, severidad y el rendimiento agronómico. Las diferencias entre tratamientos fueron significativas indicando la importancia epidemiológica de la semilla infectada. Se concluye que la mezcla de iprodione + triticonazole fue eficiente en el control de $D$. teres en semilla, in vitro y a campo, permitiendo disminuir significativamente la epidemia de la mancha en red.

Palabras claves: Pyrenophora teres, Hordeum distichum, patógeno de semilla, curasemillas.

\section{ABSTRACT \\ Effect of iprodione and its mixtures with thiram and triticonazole to control Drechslera teres in barley seed}

Net blotch is the most important barley (Hordeum vulgare) disease in Argentina. The seed chemical treatment is an important measure to avoid the introduction and dispersion of $D$. teres. The objectives of the present study were: 1) to evaluate in vitro the fungicide iprodione in different dosages and its mixtures with triticonazole and thiram and to compare them to the most common fungicides used (tebuconazole and flutriafol), and 2) to study under field conditions, the best treatment achieved in vitro. Seed treatment studies indicated that the fungicides used in Argentina such as tebuconazole and flutriafol were less effective against $D$. teres than iprodione $(50 \mathrm{~g}$ i.a.), iprodione + triticonazole, and iprodione + thiram. The number of focuses of primary infection, incidence, severity, and agronomic yield in paired plots with iprodione + triticonazole and without fungicide were measured in field. Differences in the valued of these variables were significant among parcels indicating the importance of seed infection in the epidemiology of the disease. It concludes that the mixture of iprodione + triticonazole was effective against $D$. teres both in vitro and un the field significantly decreasing the incidence of net blotch epidemic.

\section{INTRODUCCION}

El cultivo de cebada cervecera (Hordeum vulgare L.) es actualmente, uno de los más importantes en Argentina debido a su gran expansión experimentada en los últimos años como consecuencia de la exportación de malta a Brasil, de la integración del MERCOSUR y del aumento del consumo interno de la cerveza. Entre los factores limitantes del cultivo figuran las enfermedades fúngicas. Los datos de los registros epifitiológicos han revelado que la «mancha en red» causada por Drechslera teres (Sacc.) Shoen, es una enfermedad endémica, la de mayor prevalencia ( $76,0 \%$ sobre 117 campos examinados en 1990/93) e incidencia (100,0\% en 12 campos sobre 25 examinados en 1991) presentándose desde el estado de plántula a cosecha (Carmona et al., 1992; Carmona, 1994a; Barreto \& Carmona, 1993, 1994a, 1996). A su vez, los monitoreos de la sanidad de semilla indicaron ser este el principal patógeno. En 1991 y 1993, los promedios y máximos valores registrados en semilla fueron: $13,5 \%$ y $35,0 \%$ y $11,0 \%$ y $57,0 \%$ respectivamente (Barreto \& Carmona, 1993). Los 
Efecto del fungicida iprodione y sus mezclas con thiram y...

daños en el rendimiento causados por la enfermedad estimados en 1994, fueron del 20,0\%; representando $733 \mathrm{Kg} / \mathrm{ha}$ (Carmona, 1994b).

La asociación de este patógeno necrotrófico con la semilla representa un mecanismo eficaz y seguro para garantizar la continuidad de su ciclo biológico entre cosechas. Por ello, el tratamiento de semillas constituye una importante medida de control para impedir la introducción del patógeno donde se siembra cebada por primera vez o la re-introducción en aquéllos donde se practica la rotación de cultivos. Sin embargo, en Argentina existen muy pocos estudios sobre el control químico de $D$. teres en semillas de cebada (Barreto \& Carmona, 1994b).

Trabajos de investigadores, (Reis \& Forcelini, 1993; Reis et al., 1995 y 1997; Forcelini, 1992, Barreto \& Carmona, 1994b) reportan que el iprodione es eficiente fungicida para D. teres como también para Bipolaris sorokiniana (Sacc. in Sorok).

En Argentina el iprodione y el triticonazole son principios activos de reciente registro para semillas. Por ello, ante la imperiosa necesidad de obtener información, se llevó a cabo este trabajo cuyos objetivos fueron evaluar in vitro la fungitoxicidad del fungicida iprodione en diferentes dosis y sus mezclas con thiram y triticonazole comparando su efecto con los fungicidas más usados en Argentina (tebuconazole y flutriafol), y valorar a campo el mejor tratamiento.

\section{MATERIALES Y METODOS}

\section{Evaluación de la fungitoxicidad}

En este ensayo se utilizó una muestra de semillas del cultivar Quilmes Pampa cosechada en 1996 proveniente de un lote que mostró severos síntomas de la mancha en red. Se utilizaron $300 \mathrm{~g}$ de semilla por tratamiento. Los fungicidas y dosis utilizadas en g i.a./100 Kg. de semilla, fueron las siguientes: iprodione $25 \mathrm{~g}$ y $50 \mathrm{~g}$; iprodione + triticonazole $(25 \mathrm{~g}+2,5 \mathrm{~g})$; iprodione + triticonazole $(50 \mathrm{~g}+2,5 \mathrm{~g})$; iprodione + thiram $(50 \mathrm{~g}+150 \mathrm{~g})$; tebuconazole $2,5 \mathrm{~g}$ y flutriafol $5 \mathrm{~g}$. Una muestra sin tratar fue considerada como testigo.

Las semillas fueron tratadas vía húmeda al 2,0\% de agua en erlenmeyers de $500 \mathrm{ml}$. Cada tratamiento fue agregado gradualmente agitando las semillas durante $10 \mathrm{~min}$ para lograr una cobertura uniforme. Después del tratamiento, las semillas (10/caja) fueron plaqueadas en cajas de Petri de vidrio conteniendo medio semi-selectivo para Cochliobolus sativus (Ito \& Kurib) Dreschsk. ex Dastur (Reis, 1983) por ser éste un excelente medio para detectar a $D$. teres (Carmona et al., 1999)

Las cajas fueron incubadas en cámara climática con fotoperíodo $12 \times 12 \mathrm{~h}$, con luz cercana al ultravioleta (NUV) y temperatura entre $20^{\circ} \mathrm{C}-23^{\circ} \mathrm{C}$. Luego de 10 días se procedió al análisis de las semillas con microscopio estereoscópico (40 $\mathrm{X}$ ) considerando semilla infectada a aquélla donde el hongo produjo conidióforos con conidios. El diseño estadístico fue completamente aleatorizado con cuatro repeticiones de 50 semillas por tratamiento. Los datos fueron expresados como porcentaje de semillas infectadas y porcentaje de control. Finalmente los datos fueron transformados $\mathrm{a} \sqrt{x+0.5} \mathrm{y}$ analizados estadísticamente mediante análisis de varianza y test de Tukey al 5,0\% para diferenciar las medias.

\section{Experimento a campo}

El ensayo fue conducido en el campo experimental de la empresa Maltería Pampa en Coronel Suárez $(560$ km) al sur de Buenos Aires durante 1996. Para trabajar con la semilla como fuente exclusiva de inóculo, se seleccionó un lote donde no se había sembrado cebada anteriormente. La muestra utilizada fue del cultivar Quilmes Pampa con una incidencia de $64,0 \%$.

El tratamiento de las semillas fue realizado de manera similar a la descripta anteriormente, pero utilizando $500 \mathrm{~g} /$ tratamiento. Una muestra sin tratar fue considerada como testigo.

Las semillas tratadas fueron sembradas con sembradora en pequeñas parcelas de siete hileras y de seis metros de longitud (110 g de semilla/parcela). El diseño experimental fue el de parcelas apareadas con cuatro repeticiones. Para evitar el efecto entre tratamientos, se sembró entre parcelas de cebada y entre bloques, un hospedante no susceptible (trigo, cv. Buck Yapeyú) en parcelas de seis hileras $\mathrm{y}$ de $6 \mathrm{~m}$ de longitud.

A los 30 días de la siembra se evaluó la fitotoxicidad de cada tratamiento mediante el conteo de plantas normales emergidas en dos hileras al azar para cada parcela. Los datos fueron expresados como número de plantas emergidas/metro .En esa misma evaluación también se determinó el número de plántulas enfermas considerando como tal, a aquéllas que presentaron al menos una mancha típica de la mancha en red. Los datos fueron expresados como incidencia (I, porcentaje de plántulas enfermas). Al estado de hoja bandera, se determinó el porcentaje de hojas bandera enfermas en cada parcela (I) y el porcentaje de área foliar necrosada de estas mismas hojas ( $\mathrm{S}$, severidad) sobre un total de 10 plantas al azar extraídas de cada repetición. Para expresar la (S) en cada parcela, los porcentajes del área foliar necrosada de cada hoja bandera fue sumada y dividido por el total de hojas bandera examinadas.

A la cosecha se determinó el rendimiento manualmente sobre una superficie central de $4,2 \mathrm{~m}^{2}$ de cada parcela, excluyendo las borduras.

Para el análisis estadístico los datos de porcentaje fueron transformados a $\sqrt{X+0,5}$ los promedios comparados con el test " $t$ ". Los porcentajes de control para cada evaluación, fueron determinados en relación al testigo

\section{RESULTADOS Y DISCUSION}

\section{Fungitoxicidad específica}

El análisis estadístico de los valores obtenidos muestra que todos los tratamientos químicos difirieron significativamente $(\mathrm{p}<0,05)$ del testigo (Tabla 1$)$. 


\section{A. Carmona et al.}

TABLA 1 - Efecto de diferentes fungicidas sobre el control de Drechslera teres en semillas de cebada (Hordeum vulgare). 1996

\begin{tabular}{lccc}
\hline \hline Tratamiento & $\begin{array}{c}\text { Dosis: g de } \\
\text { ingrediente ativo/ } \\
\mathbf{1 0 0 ~ K g ~ d e ~ s e m i l l a s ~}\end{array}$ & $\begin{array}{c}\text { Incidencia en } \\
\text { semilla (\%) }\end{array}$ & $\begin{array}{c}\text { Contro } \\
\mathbf{( \% )}\end{array}$ \\
\hline 1.Testigo & -- & $89,5 \mathrm{e}^{\mathrm{l}}$ & -- \\
2.Iprodione + Thiram & $50+150$ & $2,5 \mathrm{a}$ & 97,2 \\
3.Iprodione + Triticonazole & $50+2,5$ & $3 \mathrm{a}$ & 96,6 \\
4.Iprodione & 50 & $4 \mathrm{a}$ & 96,5 \\
5.Iprodione + Triticonazole & $25+2,5$ & $11,5 \mathrm{~b}$ & 87 \\
6. Iprodione & 25 & $27,5 \mathrm{c}$ & 69,2 \\
\hline 7.Tebuconazole & 2,5 & $33 \mathrm{c}$ & 63 \\
\hline 8.Flutriafol & 5 & $48,5 \mathrm{~d}$ & 45,8 \\
\hline
\end{tabular}

* Datos transformados a $\sqrt{x+0.5}$

${ }^{1}$ Letras iguales no muestran diferencias significativas según Test de Tukey $(\mathrm{p}<0.05), \mathrm{CV}(\%) 22,3$

Los resultados muestran que el iprodione a $50 \mathrm{~g}$ i.a. o mezclado con thiram o con triticonazole redujeron la incidencia en las semillas y fueron, en control, superior a los dos fungicidas triazólicos más utilizados y a los demás tratamientos. En relación al iprodione, la dosis de $25 \mathrm{~g}$ i.a. sólo o en mezcla con el triticonazole resultaron ineficaces para el control de $D$. teres. Estos resultados coinciden con los obtenidos en estudios preliminares (Barreto \& Carmona, 1994). La poca eficiencia de estos fungicidas explicarían la transmisión de $D$. teres en aquéllos campos nuevos o bajo rotación, como fueron observados en relevamientos a campo en años anteriores (Carmona et al., 1992; Barreto \& Carmona, 1996).

En relación a la mezcla de iprodione $50 \mathrm{~g}+$ triticonazole $2.5 \mathrm{~g}$ es de destacar que no se diferenció significativamente del iprodione sólo o en su mezcla con thiram. Estos resultados, los comienzos de la inscripción de ambos productos como curasemilla en la Argentina y la poca disponibilidad de semilla, determinaron que fuera el tratamiento llevado para el ensayo a campo.

\section{Experimento a campo}

El mayor porcentaje de control y el mayor rendimiento fue obtenido con el tratamiento químico de la semilla sin afectar la emergencia de plantas/m comparado con el testigo (Tabla 2). Por eso al analizar el número de plantas/m emergidas, no existieron diferencias significativas entre el testigo y el tratamiento químico.

El porcentaje de plántulas enfermas en el testigo alcanzó el 15,0\%, mientras que en tratado fue de sólo 3,0\% $(\mathrm{p}<0,05)$. El porcentaje de control fue $80,0 \%$ lo que demuestra que la mezcla seleccionada demostró una elevada fungitoxicidad. Al estado de hoja bandera, se observó un rápido crecimiento de la enfermedad en el testigo alcanzando una I del $85,0 \%$, respecto al $15,0 \%$ observado en el tratado $(\mathrm{p}<0,05)$. Esto significó un $82,0 \%$ de control.

En relación a la $\mathrm{S}$ determinada sobre las hojas bandera, alcanzó un 8,0\% en la parcela testigo y sólo 1,0\% en la mezcla $(\mathrm{p}<0,05)$ determinando un elevado porcentaje de control $(87,0 \%)$.
TABLA 2 - Efecto a campo del iprodione $(50$ g p.a) + triticonazole (2.5 g p.a.) Plantas $/ \mathrm{m}$, incidencia en plántulas, incidencia (I) y severidad (S) en hojas banderas, porcentajes de control y rendimientos del ensayo. Cnel Suárez. 1996

\begin{tabular}{lccc}
\hline \hline \multirow{2}{*}{ Evaluación } & \multicolumn{2}{c}{ Tratamientos* } & $\begin{array}{c}\text { \% de control } \\
\text { respecto al } \\
\text { testigo }\end{array}$ \\
\cline { 2 - 3 } & Testigo & $\begin{array}{c}\text { Trticonazole 2,5 g i.a. } \\
+ \text { iprodione 50 g i.a. }\end{array}$ & -- \\
\hline Emergencia & $39 \mathrm{a}$ & $40 \mathrm{a}^{1}$ & 79,6 \\
I en plántuh (\%) & $14,75 \mathrm{a}$ & $3 \mathrm{~b}$ & 82 \\
I en hoja bandera (\%) & $85 \mathrm{a}$ & $15 \mathrm{~b}$ & 87 \\
\hline S en hoja bandera $(\%)$ & $8,07 \mathrm{a}$ & $1,07 \mathrm{~b}$ & -- \\
\hline Rendimientos $(\mathrm{Kg} / \mathrm{ha})$ & $2848 \mathrm{a}$ & $3429 \mathrm{~b}$ & \\
\hline
\end{tabular}

* Datos transformados a $\sqrt{x+0.5}$. Promedio de cuatro repeticiones

${ }^{1}$ Letras iguales entre columnas no difieren entre sí Test " $\mathrm{t}$ " al 5,0 \%.

Respecto al rendimiento agronómico, el tratamiento químico al controlar la enfermedad, posibilitó que el cultivar expresase su rendimiento de granos $21,0 \%$ superior al rendimiento del testigo, lo que representó un aumento de 521 $\mathrm{kg} / \mathrm{ha}$.

De acuerdo con los dos experimentos evaluados, la mezcla de iprodione + triticonazole demostró ser eficiente en el control de $D$. teres de la semilla bajo condiciones in vitro y a campo, permitiendo controlar la epidemia de la mancha en red. Asimismo el ensayo a campo también logró confirmar la importancia epidemiológica de la semilla infectada por $D$. teres. La cuantificación de la enfermedad en las parcelas testigo ponen de manifiesto la eficiente transmisión del patógeno indicando la importancia de la semilla infectada como fuente de inóculo primario (Hampton,1980). La inexistencia de restos culturales infestados y la pequeña separación de las parcelas de $1 \mathrm{~m}$ de ancho, permiten corroborar que la semilla constituyó la única fuente de inóculo y que probablemente, el viento no participó en la diseminación del mismo.

Bajo las condiciones de este ensayo un daño de 521 $\mathrm{kg} / \mathrm{ha}$ puede ser directamente atribuida a la siembra de semillas enfermas. De acuerdo con los resultados obtenidos, es necesario replantearse los objetivos del tratamiento curasemilla en cebada. Actualmente la casi totalidad de los fungicidas utilizados en semillas de cebada, están prioritariamente dirigidos a controlar el carbón volador [Ustilago nuda (Jens.) Kell. y Swing] enfermedad que es de carácter secundario como fue demostrado en monitoreos anteriores (Carmona et al., 1992). El control del carbón debe estar dirigido principalmente a los lotes destinados a semilla (Galich \& Galich, 1994).

Por eso, se debe orientar el uso de los curasemillas contra $D$. teres, inductor de la mancha en red que es actualmente la principal enfermedad de los cultivos de cebada en Argentina.

Nuevos estudios de la importancia epidemiológica de la semilla infectada y principios activos y/o coadyuvantes orgánicos que puedan controlar eficientemente a los agentes causantes de manchas foliares, deberían ser implementados 
Efecto del fungicida iprodione y sus mezclas con thiram y...

\section{AGRADECIMIENTOS}

Los autores agradecen a Maltería Pampa S.A. y a sus Ings. Mario Cattáneo y Adrián Schmidt por el apoyo técnico recibido, y a la Srta. María Rosa Gonzalez (INTA Castelar) por la asistencia prestada.

\section{REFERENCIAS BIBLIOGRAFICAS}

BARRETO, D.E. \& CARMONA, M.A. Microflora of barley seed and malt in Argentina. Proceedings 1 International Seed Testing Association, Plant Disease Committee. Symposium on Seed Health Testing, Ottawa, Canada, 1993. p.70-74.

BARRETO, D.E. \& CARMONA M.A. Panorama sanitario de la cebada cervecera Argentina. Estado actual de las investigaciones 1990-1993. Actas de las 1ras. Jornadas de actualización técnica-económica del cultivo de cebada cervecera. Bolsa de cereales, Buenos Aires. 1994a. p. 100-106.

BARRETO, D.E. \& CARMONA, M.A. Eficiencia in vitro de fungicidas en tratamiento de semillas para el control de Drechslera teres. Acta 3 Congreso Nacional de Trigo y I Simposio Nacional de Cereales de Siembra OtoñoInvernal, Bahía Blanca, Argentina.1994b. p. 289-290.

BARRETO, D.E. \& CARMONA, M.A. Field incidence of Pyrenophora teres and infection on barley seed. Revista de Investigaciones Agropecuarias 26:61-65.1996.

CARMONA, M.A., BARRETO, D.E. \& WRIGHT, E.R. Relevamiento de enfermedades fúngicas de la cebada cervecera en la provincia de Buenos Aires en 1990. Revista de la Facultad de Agronomía 13:187-192. 1992.

CARMONA, M. A. Distribución, incidencia y severidad de las enfermedades foliares de la cebada cervecera en la provincia de Buenos Aires en 1992. Fitopatología 29:214-217. 1994a.
CARMONA, M.A. Informe de avance de Beca de Investigación y Perfeccionamiento (1993/94). La mancha en red de la cebada. Manejo y estimación de pérdidas. Secretaría de Ciencia y Técnịca. Facultad de Agronomía, Universidad de Buenos Aires, 1994b.

CARMONA, M.A. \& BARRETO, D.E. Enfermedades fúngicas de la cebada cervecera en la provincia de Buenos Aires (Argentina) en 1991. Fitopatologia Brasileira 20:509-510. 1995.

CARMONA, M.A., BARRETO, D.E. \& REIS, E.M. Detection, transmission and control of Drechslera teres in barley seed. Seed Science \& Technology 27:761-769. 1999.

FORCELINI, C.A. Incidência, transmissão e controle de Bipolaris sorokiniana em sementes de trigo. (Tese de Mestrado). São Paulo. Universidade de São Paulo.1992.

GALICH, A \& GALICH, M. No calque el '93, Quítele el Carbón, Clarín Rural. 1994.

HAMPTON, J. G. The role of seed borne inoculum in the epidemiology of net blotch of barley in New Zealand. New Zealand Journal of Experimental Agriculture. 8:297-299. 1980.

REIS, E.M. Selective medium for isolating Cochliobolus sativus from soil. Plant Disease 67:68-70.1983.

REIS, E.M. \& FORCELINI, C.A. Transmissão de Bipolaris sorokiniana de sementes para órgãos radiculares e aéreos do trigo. Fitopatologia Brasileira 18:76- 81.1993.

REIS, E.M., CASA TREZZI, R. \& SILVA, M.S. Efeito do tratamento de sementes de cevada no controle e no desenvolvimento da mancha em rede, causada por Drechslera teres. Fitopatologia Brasileira 20:561-565. 1995.

REIS, E.M., CASA, M., CASA TREZZI, R., CARMONA, M.A. \& BARRETO, D.E. Sensibilidade de Drechslera teres ao fungicida triadimenol usado em tratamento de sementes de cevada. Fitopatologia Brasileira 22:539-543. 1997. 